\title{
Optical force rectifiers based on PT-symmetric metasurfaces
}

\author{
Rasoul Alaee, ${ }^{1, *}$ Burak Gurlek, ${ }^{1}$ Johan Christensen, ${ }^{2}$ and Muamer Kadic ${ }^{3}$ \\ ${ }^{1}$ Max Planck Institute for the Science of Light, Erlangen 91058, Germany \\ ${ }^{2}$ Universidad Carlos III de Madrid, ES-28916 Leganés, Madrid, Spain \\ ${ }^{3}$ Institut FEMTO-ST, UMR 6174, CNRS, Université de Bourgogne Franche-Comté, 25000 Besançon, France
}

(Received 27 November 2017; revised manuscript received 27 April 2018; published 14 May 2018)

\begin{abstract}
We introduce here the concept of optical force rectifier based on parity-time symmetric metasurfaces. Directly linked to the properties of non-Hermitian systems engineered by balanced loss and gain constituents, we show that light can exert asymmetric pulling or pushing forces on metasurfaces depending on the direction of the impinging light. This generates a complete force rectification in the vicinity of the exceptional point. Our findings have the potential to spark the design of applications in optical manipulation where the forces, strictly speaking, act unidirectionally.
\end{abstract}

DOI: 10.1103/PhysRevB.97.195420

\section{INTRODUCTION}

Light carries energy and momentum [1,2] and its interaction with matter will exert an optical force in the direction of the light flow. The optical pushing force was first introduced by Johannes Kepler in 1619 and is known as radiation pressure. The magnitude of the radiation pressure is directly related to the rate of change of the momentum. This radiation pressure leads to many practical applications in astronomy, astrodynamics, and cavity-optomechanical systems. Solar and microwave sails as well as ambitious projects such as laser-propelled spaceships are excellent examples where the radiation pressure is used to drive a sailcraft [3].

For passive objects, the radiation pressure force exerted by a plane wave is always pushing or zero and limited to $0 \leqslant F \leqslant \frac{2 I_{0}}{c}\left(I_{0}\right.$ is the intensity of the incident light and $c_{0}$ the speed of light in vacuum). The lower $\left(F_{\min }=0\right)$ and the upper $\left(F_{\max }=\frac{2 I_{0}}{c}\right)$ bounds correspond to transparent objects and perfect mirrors, respectively [2]. For active objects, however, it has been shown that the direction of the exerted optical force can be reversed, which leads to a counterintuitive force known as the optical pulling force [4-6]. An alternative for passive objects to explore such optical pulling force is realized by using, e.g., a tractor beam, i.e., a Bessel beam, a solenoid beam, or a superposition of plane waves [7-12].

Artificial materials such as metamaterials and metasurfaces have been used to fully control and manipulate the flow of reflected and transmitted light by breaking the time reversal symmetry or the parity symmetry [13-18]. In particular, metasurfaces without parity symmetry, i.e., broken inversion symmetry, have been used to control the reflected light [17-20]. By breaking the Lorentz reciprocity (i.e., applying an external magnetic field, using nonlinear materials, or time-dependent permittivities), one can control the transmitted light and obtain unidirectional wave propagation [21].

*Corresponding author: rasoul.alaee@gmail.com
Recently, inspired by parity-time (PT) symmetric quantum mechanics, a new class of artificial optical systems have been proposed to control the scattering of light [23]. These PT-symmetric optical systems are invariant under combined parity and time inversion and can be obtained by introducing balanced gain and loss where the refractive index should satisfy $n(\mathbf{r})=n^{*}(-\mathbf{r})$ [24-27]. The concept of PT symmetry in optics led to several interesting optical phenomena such as asymmetric propagation [28], unidirectional invisibility [29], optical lasing [26], optical sensing [30], wireless power transfer [31], and many others.

An interesting question, which has not yet been fully addressed in the literature [32], is what kind of optical forces can be achieved in PT-symmetric metasurfaces? Is it possible to design optical force rectifiers, i.e., asymmetric one-way optical forces?

In this paper, we propose an optical force rectifier based on PT-symmetric meta-atoms (i.e., coupled spheres) [see Figs. 1(a) and 1(b)] and metasurfaces composed of arrays of such meta-atoms [see Fig. 4]. We find a direct link between the PT-phase transition and the asymmetric optical pulling, pushing, or zero force when illuminated at opposite directions.

\section{OPTICAL FORCE ON DIPOLAR PARTICLES}

To understand the underlying physics of the optical forces exerted on PT-symmetric particles (made of balanced gain and loss), we first study the optical forces in connection with individual passive (lossless or lossy) and active (gain) particle. Under the dipolar approximation, the time-averaged exerted force on an isotropic particle by an arbitrarily polarized plane wave propagating in $\mathbf{e}_{k}$ direction is given by [33-35]

$$
\mathbf{F}=\left(F_{p}+F_{m}+F_{p m}\right) \mathbf{e}_{k},
$$

which has three different contributions, i.e., the electric $F_{p}=$ $F_{p}^{\max } \operatorname{Im}\left(\alpha_{e} / \alpha_{0}\right)$, the magnetic $F_{m}=F_{p}^{\max } \operatorname{Im}\left(\alpha_{m} / \alpha_{0}\right)$, and the interference between the both $F_{p m}=-F_{p}^{\max } \operatorname{Re}\left(\alpha_{e} \alpha_{m}^{*} / \alpha_{0}^{2}\right)$. $F_{p}^{\max }=\frac{I_{0}}{c} C_{\mathrm{ext}}^{\max }$ is the universal limit on the force exerted 

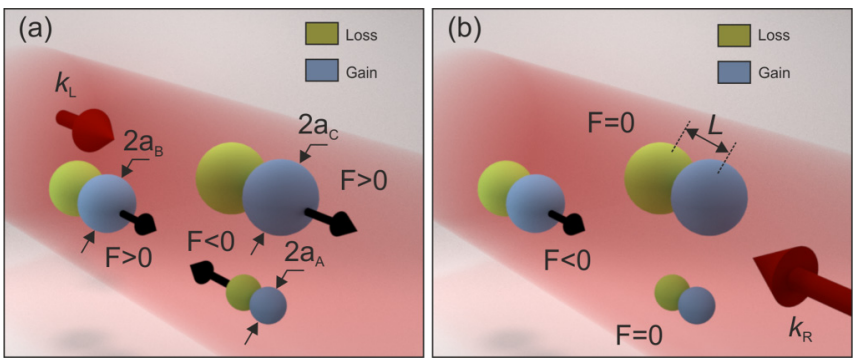

FIG. 1. Principle of an optical PT-symmetric rectifier: (a) The optical force exerted on coupled spheres by a linearly polarized plane wave from left, i.e., $k_{L}$. (b) The same as (a) for the opposite illumination direction, i.e., right $k_{R}$. We depict three cases, e.g., the coupled spheres pulled/pushed for one illumination direction (see $a_{B}$ or $a_{C}$ ) whereas zero force was exerted on the other direction, respectively. The three cases are selected after the design explained in Fig. 3. $a$ indicates the radius, $L=2.6 a$ the distance between two spheres, and $F$ the resulting optical force. The coupled spheres are assumed to be connected by a lossless dielectric with a refractive index of the embedding medium (see the Supplemental Material [22] for an alternative structure made of coupled disks).

on an electric dipole [35], $\alpha_{0}=6 \pi / k^{3}$ and $C_{\mathrm{ext}}^{\max }=\frac{3 \lambda^{2}}{2 \pi}$ are the maximum extinction cross section of an electric dipolar particle [35,36]. $\alpha_{e}$ and $\alpha_{m}$ are the electric and magnetic polarizabilities of the particle, respectively. $I_{0}=\frac{1}{2} \epsilon_{0} c\left|E_{0}\right|^{2}$ is the intensity of the illumination, $k$ the wave number, and $\epsilon_{0}$ the free space permittivity.

\section{A. Passive particles}

The exerted optical force on an isotropic passive particle is always positive (pushing) [35]. Figures 2(a) and 2(d) show the normalized optical force exerted by a linearly polarized plane wave on a sphere as a function of the sphere' s size parameter $a / \lambda$ and the refractive index ratio $n^{\prime \prime} / n^{\prime}$. The optical force is calculated for two cases: a dual particle, i.e., $\epsilon_{r}=$ $\mu_{r}$ [see Figs. 2(a)-2(c)] [37], and a particle with $\mu_{r}=1$ [see Figs. 2(d)-2(f)]. For a passive sphere $\left(n^{\prime \prime} \geqslant 0\right)$, it can be seen that the force is always pushing, i.e., along the propagation direction of the impinging light $\mathbf{e}_{k}$. This can be explained from the fact that the electric $F_{p}$ and magnetic $F_{m}$ forces are always positive for the passive particle $\left(n^{\prime \prime} \geqslant 0\right)$ because $\operatorname{Im}\left(\alpha_{e}\right)$ and $\operatorname{Im}\left(\alpha_{m}\right)$ are positive for all wavelengths [see Fig. 2(c)], whereas the interference force contribution $F_{p m}$ (positive or negative) is small compared to $F_{p}$ and $F_{m}$. Thus, the total force is always pushing (non-negative) [see Fig. 2(c)]. Note that the inverse of the polarizabilities satisfy

$$
\operatorname{Im}\left(\alpha_{0} / \alpha_{e}\right)=-1, \text { and } \operatorname{Im}\left(\alpha_{0} / \alpha_{m}\right)=-1,
$$

for isotropic lossless particles [see point M in Figs. 2(b) and 2 (e) and the vertical black dashed line]. These relations can be obtained from the conservation of the energy $[1,38]$. It can be seen that for a lossy particle, the $\operatorname{Im}\left(\frac{\alpha_{0}}{\alpha_{e} / m}\right)<-1$ due to the Ohmic losses [see the green area in Figs. 2(b) and 2(e)].
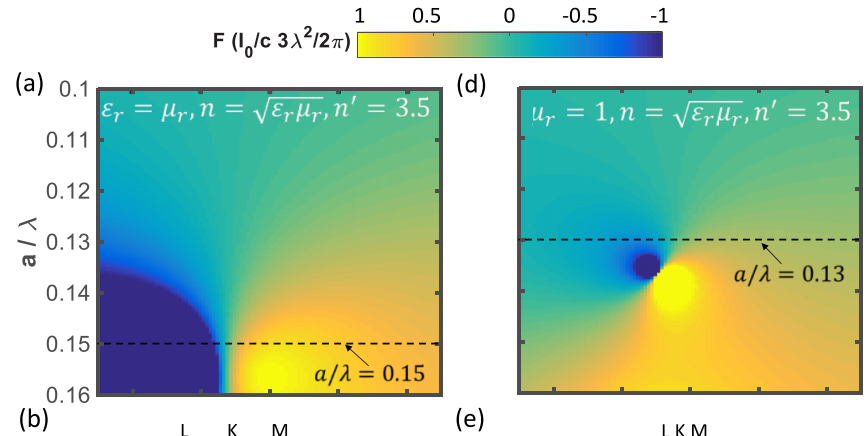

(e) LKM
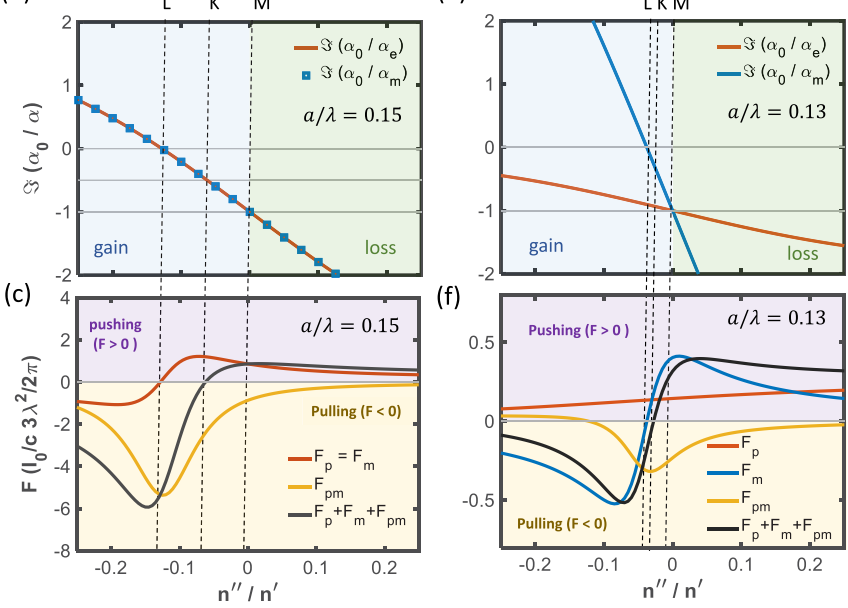

FIG. 2. Single sphere: (a) The normalized optical force exerted by a linearly polarized plane wave on a dual sphere as a function of the sphere's size parameter $(a / \lambda, a$ is the radius of sphere) and the refractive index ratio $\left(n^{\prime \prime} / n^{\prime}, n=n^{\prime}+i n^{\prime \prime}, n=\sqrt{\epsilon_{r} \mu_{r}}\right.$ is the refractive index of the dual sphere, i.e., $\epsilon_{r}=\mu_{r}$ ). (b) The imaginary part of the inverse of electric and magnetic polarizabilities as a function of the the refractive index ratio at $a / \lambda=0.15$, where $a$ is the radius of the sphere. (c) The normalized optical force exerted by a linearly polarized plane wave on a dual sphere as a function the refractive index ratio. (d)-(f) The same as (a)-(c) for a sphere with $\mu_{r}=1$ and for (e)-(f) size parameter is $a / \lambda=0.13$. The optical force and polarizabilities normalized to $\frac{3 \lambda^{2}}{2 \pi} \frac{I_{0}}{c}$ and $\alpha_{0}=\frac{6 \pi}{k^{3}}$, respectively. The real part of the refractive index is fixed $n^{\prime}=3.5$. All figures have the same $x$ axis.

\section{B. Active particles}

Let us now consider active particles. The imaginary part of the inverse of polarizabilities satisfy $\operatorname{Im}\left(\frac{\alpha_{0}}{\alpha_{e} / m}\right)>-1$, and by decreasing the imaginary part of the refractive index (i.e., increasing gain) $n^{\prime \prime}$, at some point the total loss will be zero, i.e., $\operatorname{Im}\left(\frac{\alpha_{0}}{\alpha_{e / m}}\right)=0$ [see the L point in Figs. 2(b) and 2(e)]. This occurs when the scattering loss is identical to the Ohmic loss. For an active particle, the optical force can be pulling. The pulling force for the active particles originates either from a large negative contribution of the interference term, i.e., $F_{p m}<$ 0 , or can be induced by the negative contribution of the electric or magnetic terms (i.e., $F_{p}<0$ and $F_{m}<0$ ), depending on the imaginary part of the refractive index, i.e., $n^{\prime \prime}$ [both in Figs. 2(c) and 2(f)]. Using Eq. (1), the optical force for a dual particle (i.e., $\mu_{r}=\epsilon_{r}, \alpha=\alpha_{e}=\alpha_{m}, F_{p}=F_{m}$ ) is simplified to

$$
\mathbf{F}=F_{p}^{\max }\left[2 \operatorname{Im}\left(\frac{\alpha}{\alpha_{0}}\right)-\frac{|\alpha|^{2}}{\alpha_{0}^{2}}\right] \mathbf{e}_{k} .
$$


The total force vanishes if $\operatorname{Im}\left(\frac{\alpha_{0}}{\alpha}\right)=-0.5$ [compare the back dashed line and see the K point in Figs. 2(b) and 2(c)] and will be pulling force for $\operatorname{Im}\left(\frac{\alpha_{0}}{\alpha}\right)>-0.5$. Similar explanations can be used to understand the exerted force on an active or a passive particle with $\mu_{r}=1$, i.e., Figs. 2(d)-2(f). Note that the gain particle can be achieved by optically pumped quantum emitters (e.g., quantum dots, dye molecules, or rare-earth ions) embedded in a lossless particle $[39,40]$. The exerted force by the pumped beam is zero when the particle is illuminated by two pumped beams with opposite illumination directions [6]. A similar pumping scheme can be used to obtain balanced gain and loss particles, i.e., PT meta-atoms.

\section{PT-SYMMETRIC META-ATOMS}

In the following, we extend the previous cases of individual particles carrying either. In general, the exerted force on coupled dipolar particles can be analytically calculated using a coupled dipole approximation. The total time-averaged exerted force by an arbitrarily polarized plane wave propagating in $\mathbf{e}_{k}$ direction is $\mathbf{F}=\sum_{i=1}^{N} \mathbf{F}_{i}$, where $N$ is the number of particles, and $\mathbf{F}_{i}$ is the exerted force at the position of $i$ th particle placed at position $\mathbf{r}_{i}$. For dipolar particles defined as

$$
\begin{aligned}
\mathbf{F}_{i}= & \frac{1}{2} \operatorname{Re}\left\{\left[\nabla \mathbf{E}_{\mathrm{loc}}^{*}\left(\mathbf{r}_{i}\right)\right] \cdot \mathbf{p}_{i}\right\}+\frac{1}{2} \operatorname{Re}\left\{\left[\nabla \mathbf{B}_{\mathrm{loc}}^{*}\left(\mathbf{r}_{i}\right)\right] \cdot \mathbf{m}_{i}\right\} \\
& -\frac{Z_{0} k^{4}}{12 \pi} \operatorname{Re}\left(\mathbf{p}_{i} \times \mathbf{m}_{i}^{*}\right),
\end{aligned}
$$

where $\mathbf{E}_{\mathrm{loc}}\left(\mathbf{r}_{i}\right)$ and $\mathbf{H}_{\mathrm{loc}}\left(\mathbf{r}_{i}\right)$ are the local electric and magnetic fields defined as $[41,42]$

$$
\begin{gathered}
\mathbf{E}_{\mathrm{loc}}\left(\mathbf{r}_{i}\right)=\mathbf{E}_{\mathrm{inc}}\left(\mathbf{r}_{i}\right)+\mathbf{E}_{\mathrm{int}}\left(\mathbf{r}_{i}\right), \\
\mathbf{H}_{\mathrm{loc}}\left(\mathbf{r}_{i}\right)=\mathbf{H}_{\mathrm{inc}}\left(\mathbf{r}_{i}\right)+\mathbf{H}_{\mathrm{int}}\left(\mathbf{r}_{i}\right), \\
{\left[\begin{array}{c}
\mathbf{E}_{\mathrm{int}}\left(\mathbf{r}_{i}\right) \\
\mathbf{H}_{\mathrm{int}}\left(\mathbf{r}_{i}\right)
\end{array}\right]=\mathbf{G}\left[\begin{array}{c}
\mathbf{p}_{j} \\
\mathbf{m}_{j}
\end{array}\right], \quad \mathbf{G}=\left(\begin{array}{cc}
\overline{\bar{A}} & \overline{\bar{B}} \\
\overline{\bar{C}} & \overline{\bar{D}}
\end{array}\right),} \\
\overline{\bar{A}}=\overline{\bar{G}}\left(\mathbf{r}_{i}, \mathbf{r}_{j}\right)\left(1-\delta_{i j}\right), \\
\overline{\bar{B}}=-\frac{Z_{0}}{c} \nabla \times \overline{\bar{G}}\left(\mathbf{r}_{i}, \mathbf{r}_{j}\right)\left(1-\delta_{i j}\right), \\
\overline{\bar{C}}=\nabla \times \overline{\bar{G}}\left(\mathbf{r}_{i}, \mathbf{r}_{j}\right)\left(1-\delta_{i j}\right), \\
\overline{\bar{D}}=\epsilon_{0} \overline{\bar{G}}\left(\mathbf{r}_{i}, \mathbf{r}_{j}\right)\left(1-\delta_{i j}\right),
\end{gathered}
$$

where $\overline{\bar{G}}\left(\mathbf{r}_{i}, \mathbf{r}_{j}\right)$ is the Green function (see the Supplemental Material [22]). $\mathbf{E}_{\text {inc }}\left(\mathbf{r}_{i}\right)$ and $\mathbf{E}_{\text {int }}\left(\mathbf{r}_{i}\right)$ are the incident and interaction electric fields, respectively. $Z_{0}$ is the impedance of the free space. The induced moments can be obtained by using (see the Supplemental Material [22])

$$
\left(\begin{array}{c}
\mathbf{p}_{i} \\
\mathbf{m}_{i}
\end{array}\right)=\left(\begin{array}{cc}
\frac{1}{\epsilon_{0} \alpha_{e_{i}}}-\overline{\bar{A}} & -\overline{\bar{B}} \\
-\bar{C} & \frac{1}{\alpha_{m_{i}}}-\overline{\bar{D}}
\end{array}\right)^{-1}\left(\begin{array}{l}
\mathbf{E}_{\mathrm{inc}}\left(\mathbf{r}_{i}\right) \\
\mathbf{H}_{\mathrm{inc}}\left(\mathbf{r}_{i}\right)
\end{array}\right) .
$$

To calculate the exerted optical force on the coupled spheres using Eq. (4), we first calculate the induced electric and magnetic dipoles of the coupled spheres by using the following

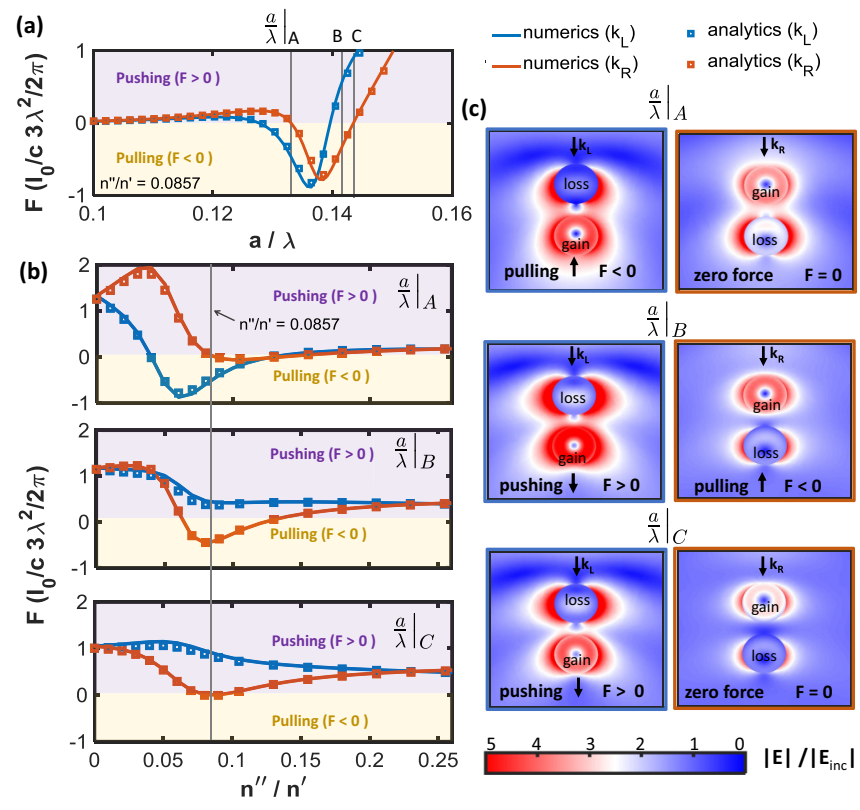

FIG. 3. The two coupled spheres with the PT symmetry: (a) The analytical (i.e., coupled dipole approximation) and numerical normalized optical force exerted by a linearly polarized plane wave on the coupled spheres for two illumination directions as a function of the single sphere' s size parameter, i.e., $a / \lambda$. The refractive index of coupled spheres are $n_{G}=n_{L}^{*}, n_{L}=n^{\prime}+i n^{\prime \prime}, n^{\prime \prime} / n^{\prime}=0.0857, n^{\prime}=$ 3.5. The blue and red colors are related to $k_{L}$ and $k_{R}$ illuminations in Figs. 1(a)-1(b), respectively. (b) The normalized optical force calculated at three different size parameters $\left.\frac{a}{\lambda}\right|_{A, B, C}$ as a function the refractive index ratio, i.e., $n^{\prime \prime} / n^{\prime}$. (c) Normalized electric fields of the coupled spheres in $x z$, plane at three different $\frac{a}{\lambda}$ for different illumination directions.

expressions $[43,44]$ :

$$
\begin{aligned}
p_{\beta}= & -\frac{1}{i \omega}\left\{\int d^{3} \mathbf{r} J_{\beta}^{\omega} j_{0}(k r)\right. \\
& \left.+\frac{k^{2}}{2} \int d^{3} \mathbf{r}\left[3\left(\mathbf{r} \cdot \mathbf{J}_{\omega}\right) r_{\beta}-r^{2} J_{\beta}^{\omega}\right] \frac{j_{2}(k r)}{(k r)^{2}}\right\}, \\
m_{\beta}= & \frac{3}{2} \int d^{3} \mathbf{r}\left(\mathbf{r} \times \mathbf{J}_{\omega}\right)_{\beta} \frac{j_{1}(k r)}{k r},
\end{aligned}
$$

where $\beta=x, y, z$, and $J_{\beta}^{\omega}$ is the induced induced electric current density is obtained by using $\mathrm{J}_{\omega}(\mathbf{r})=-i \omega \epsilon_{0}\left(\epsilon_{r}-1\right) \mathrm{E}_{\omega}(\mathbf{r})$, and $\mathrm{E}_{\omega}(\mathbf{r})$ is the electric field. We applied a numerical finite element solver (COMSOL Multiphysics) to obtain the electric field distributions. Using Eqs. (10), we numerically calculate the induced electric and magnetic dipole moments for both illumination directions [see Figs. 1(a)-1(b)]. The results are also compared with the analytical approach, i.e., coupled dipole approximation from Eq. (9), which shows a very good agreement (see Fig. 1 of the Supplemental Material [22]). By using the induced multiple moments and Eq. (4), we have analytically calculated the optical force on the coupled spheres [Fig. 3(a)] and the analytical result fits perfectly with a full-wave simulation using COMSOL Multiphysics. Note that the numerical results obtained by integrating Maxwell' s stress tensor $\overline{\overline{\mathbf{T}}}$ (see the Supplemental Material [22]) [1], i.e., 
$\mathbf{F}=\oint_{S} \overline{\overline{\mathbf{T}}} \cdot \mathbf{n} d a$, where $S$ is a surface enclosing the particle and $\mathbf{n}$ is the normal unit vector to $S$.

Figure 3(a) shows that the exerted optical force depends on the direction of the impinging light. This is due to the fact that the system does not have the mirror symmetry. As a result, the induced multipole moments are also considerably different when excited with two illumination directions (see Fig. 1 of the Supplemental Material [22]). In other words, the system is bianisotropic due to the broken inversion symmetry [38].

At the particle size parameter $\left.\frac{a}{\lambda}\right|_{A}$, and $\left.\frac{a}{\lambda}\right|_{C}$, the coupled particles are pulled/pushed for one illumination direction whereas zero force is exerted on the other direction, respectively. At the particle size parameter $\left.\frac{a}{\lambda}\right|_{B}$, the coupled particles are pulled at one illumination direction whereas they are pushed on the other direction. The field distributions for three size parameters $\left.\frac{a}{\lambda}\right|_{A, B, C}$ are plotted in Fig. 3(c). To understand the effects of loss and gain, we computed the optical force as a function of the refractive index ratio, i.e., $n^{\prime \prime} / n^{\prime}$ for these three wavelengths. For $n^{\prime \prime}=0$, the force is identical for both illuminations because the system is symmetric for both illuminations. Note that the scattering response of coupled spheres is inherently nonHermitian due to the scattering loss (even without loss or gain) and cannot be a real PT-symmetric system $[45,46]$. Therefore, we only study the PT-symmetric phase transition (and its relation with the optical optical pulling, pushing, or zero force) for the metasurface.

\section{OPTICAL FORCE ON PT-SYMMETRIC METASURFACES}

Let us consider a metasurface made of coupled spheres depicted in Fig. 4. The scattering matrix of the system can be defined as $\mathbf{S}=\left(\begin{array}{cc}t & r_{L} \\ r_{R} & t\end{array}\right)$ [47]. For the PT-symmetric metasurface, the system is reciprocal, i.e., $t=t_{R}=t_{L}$ and the unitary relation will be modified, i.e., $|T-1|=\sqrt{R_{R} R_{L}}$. This relation is known as generalized unitarity relation (GUR) [48]. $T=|t|^{2}$ is the transmittance and $R_{R / L}=\left|r_{R / L}\right|^{2}$ is the reflectance for impinging light from right/left. The eigenvalues of the scattering matrix by using $r_{L} r_{R}=t^{2}(1-1 / T)$ is given by [48]

$$
s_{1,2}=t \pm \sqrt{r_{L} r_{R}}=t(1 \pm \sqrt{1-1 / T}) .
$$

The optical force exerted per unit surface area on the metasurface by a linearly polarized plane wave at normal incidence is given by $\mathbf{F}_{R / L}=\frac{I_{0}}{c}\left(1+R_{R / L}-T\right) \mathbf{e}_{k}$, where $\mathbf{e}_{k}$ is the propagating direction. Now by using eigenvalues of the scattering materix, i.e., Eq. (11), we investigate the optical force for the PT-symmetric metasurface and its phase transition. The PT-symmetric metasurface has two phases, namely:

Symmetric phase: This phase is accompanied by unimodular eigenvalues $\left|s_{1,2}\right|=1$, where the transmission remains $T<1$ (Fig. 4). In this phase, the forces can be expressed as $F_{R, L}=\frac{I_{0}}{c}\left(R_{R, L}+\sqrt{R_{R} R_{L}}\right)$ by using the GUR. Thus, the forces will be identical for both directions, i.e., $F_{R}=F_{L}$ when reflection from both sides are identical $R_{R}=R_{L}$ (Fig. 4). In this phase, the forces are always pushing independent of the direction of illuminations.

Broken-symmetry phase: The broken-symmetry phase is characterized with larger-than-unity transmission $T>1$. In
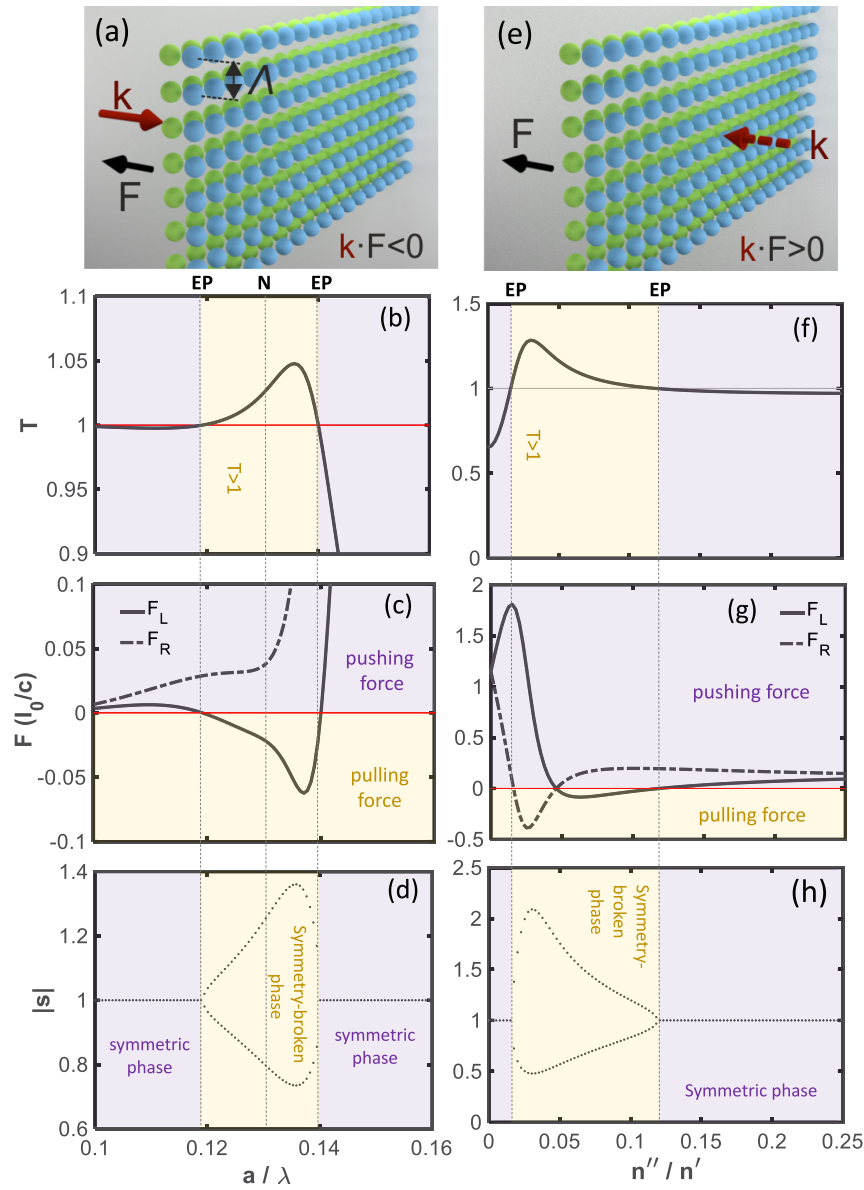

FIG. 4. PT-symmetric metasurface: (a) and (e) Schematic of the PT-symmetric metasurface. (b) and (f) Transmission spectra as a function of the single sphere' s size parameter, i.e., $a / \lambda$ and refractive index ratio, i.e., $n^{\prime \prime} / n^{\prime}$, respectively. The period in $x$ and $y$ directions are $\Lambda_{x}=\Lambda_{y}=5 a$. (c) and (g) Normalized optical force exerted on a PT-symmetric metasurface as sphere' s size parameter, i.e., $a / \lambda$ and refractive index ratio, i.e., $n^{\prime \prime} / n^{\prime}$ for both illumination directions, respectively. (d)-(h) The modulus of the eigenvalues of the $S$ matrix as sphere' s size parameter, i.e., $a / \lambda$ and refractive index ratio, i.e., $n^{\prime \prime} / n^{\prime}$. The yellow and purple shadows show the symmetry-broken and symmetric phases, respectively.

this phase, we obtain coexisting amplifying $\left|s_{1}\right|>1$ and attenuating $\left|s_{2}\right|<1$ eigenvalues (Fig. 4). Using the GUR, the force can be expressed as $F_{R, L}=\frac{I_{0}}{c}\left(R_{R, L}-\sqrt{R_{R} R_{L}}\right)$. In contrast to the PT-symmetric phase, the force always will be zero for both directions, i.e., $F_{R}=F_{L}=0$ if $R_{R}=R_{L}$ (Fig. 4). Interestingly, optical amplification and attenuation within this phase produces opposite pushing and pulling forces when illuminated from their respective directions as depicted in Fig. 4.

The transition between these two phases occurs at exceptional point (EP), i.e., unity transmission, $T=1$. At the EP, one of the reflectances vanishes (see the GUR). This will lead to a one-way optical pushing force (optical force rectifier), i.e., $F_{R}=\frac{I_{0}}{c} R_{R}$ and $F_{L}=0$ (see EPs depicted by the black dashed line in Fig. 4). Finally. it is important to mention the photophoretic force is nonzero due to the absorption in 
the investigated system. However, it is possible to suppress the photophoretic force compared to the optical force by controlling the gas pressure (see the Supplemental Material [22]) $[49,50]$.

\section{CONCLUSION}

In summary, we have shown that asymmetric optical pulling, pushing, or zero force can be achieved for PTsymmetric meta-atoms and metasurfaces when illuminated by a plane wave at opposite directions. We have found a direct physical link between the PT-symmetry phase transition and the pulling and pushing forces. Our findings might lead to applications in cavity-optomechanical systems.

\section{ACKNOWLEDGMENTS}

We thank V. Laude, D.-M. Cano, M. Wegener, and V. Sandoghdar for their constructive discussion and suggestions. R.A. and B.G. would like to acknowledge financial support from the Max Planck Society. J.C. acknowledges the support from the European Research Council (ERC) through Starting Grant No. 714577 PHONOMETA and from the MINECO through a Ramón y Cajal grant (Grant No. RYC2015-17156). This project has been performed in cooperation with the Labex ACTION program (Contract No. ANR-11LABX-0001-01) and was supported by the French Investissements d'Avenir program, Project ISITE-BFC (Contract No. ANR-15-IDEX-03).
[1] J. D. Jackson, Classical Electrodynamics (Wiley, 1999).

[2] M. Mansuripur, Field, Force, Energy and Momentum in Classical Electrodynamics (Bentham Science Publishers, United Arab Emirates, 2011).

[3] K. F. Long, Deep Space Propulsion: A Roadmap to Interstellar Flight (Springer Science \& Business Media, 2011).

[4] S. Sukhov and A. Dogariu, Opt. Lett. 35, 3847 (2010).

[5] A. Mizrahi and Y. Fainman, Opt. Lett. 35, 3405 (2010).

[6] T. Kudo and H. Ishihara, Phys. Rev. Lett. 109, 087402 (2012).

[7] S.-H. Lee, Y. Roichman, and D. G. Grier, Opt. Express 18, 6988 (2010).

[8] J. Chen, J. Ng, Z. Lin, and C. Chan, Nat. Photonics 5, 531 (2011).

[9] D. B. Ruffner and D. G. Grier, Phys. Rev. Lett. 109, 163903 (2012).

[10] D. E. Fernandes and M. G. Silveirinha, Phys. Rev. A 91, 061801 (2015).

[11] D. E. Fernandes and M. G. Silveirinha, Phys. Rev. Applied 6, 014016 (2016).

[12] E. Mobini, A. Rahimzadegan, C. Rockstuhl, and R. Alaee, arXiv:1802.01899.

[13] N. Engheta and R. W. Ziolkowski, Metamaterials: Physics and Engineering Explorations (John Wiley \& Sons, New Jersey, 2006).

[14] R. V. Craster and S. Guenneau, Acoustic Metamaterials: Negative Refraction, Imaging, Lensing and Cloaking (Springer Science \& Business Media, Dordrecht, 2012), Vol. 166.

[15] G. Milton, Extending the Theory of Composites to Other Areas of Science (Milton \& Patton Publishing, Pennsylvania, 2016).

[16] S. Mühlig, A. Cunningham, J. Dintinger, M. Farhat, S. B. Hasan, T. Scharf, T. Bürgi, F. Lederer, and C. Rockstuhl, Sci. Rep. 3, 2328 (2013).

[17] M. Odit, P. Kapitanova, P. Belov, R. Alaee, C. Rockstuhl, and Y. S. Kivshar, Appl. Phys. Lett. 108, 221903 (2016).

[18] V. S. Asadchy, A. Díaz-Rubio, S. N. Tevetkova, D.-H. Kwon, A. Elsakka, M. Albooyeh, and S. A. Tretyakov, Phys. Rev. X 7, 031046 (2017).

[19] A. Serdiukov, I. Semchenko, S. Tertyakov, and A. Sihvola, Electromagnetics of Bi-Anisotropic Materials-Theory and Application (Gordon and Breach Science Publishers, 2001), Vol. 11.

[20] R. Alaee, M. Albooyeh, A. Rahimzadegan, M. S. Mirmoosa, Y. S. Kivshar, and C. Rockstuhl, Phys. Rev. B 92, 245130 (2015).
[21] D. Jalas, A. Petrov, M. Eich, W. Freude, S. Fan, Z. Yu, R. Baets, M. Popovic, A. Melloni, J. D. Joannopoulos et al., Nat. Photonics 7, 579 (2013).

[22] See Supplemental Material at http://link.aps.org/supplemental/ 10.1103/PhysRevB.97.195420 for detailed derivations of the required relations, more examples and information on the theoretical background.

[23] F. Monticone, C. A. Valagiannopoulos, and A. Alù, Phys. Rev. X 6, 041018 (2016).

[24] R. El-Ganainy, K. G. Makris, D. N. Christodoulides, and Z. H. Musslimani, Opt. Lett. 32, 2632 (2007).

[25] C. E. Rüter, K. G. Makris, R. El-Ganainy, D. N. Christodoulides, M. Segev, and D. Kip, Nat. Phys. 6, 192 (2010).

[26] H. Hodaei, M.-A. Miri, M. Heinrich, D. N. Christodoulides, and M. Khajavikhan, Science 346, 975 (2014).

[27] B. Peng, Ş. K. Özdemir, F. Lei, F. Monifi, M. Gianfreda, G. L. Long, S. Fan, F. Nori, C. M. Bender, and L. Yang, Nat. Phys. 10, 394 (2014).

[28] S. Longhi, Phys. Rev. A 82, 031801 (2010).

[29] Z. Lin, H. Ramezani, T. Eichelkraut, T. Kottos, H. Cao, and D. N. Christodoulides, Phys. Rev. Lett. 106, 213901 (2011).

[30] H. Hodaei, A. U. Hassan, S. Wittek, H. Garcia-Gracia, R. ElGanainy, D. N. Christodoulides, and M. Khajavikhan, Nature 548, 187 (2017).

[31] S. Assawaworrarit, X. Yu, and S. Fan, Nature 546, 387 (2017).

[32] R. Alaee, J. Christensen, and M. Kadic, Phys. Rev. Applied 9 , 014007 (2018).

[33] M. Nieto-Vesperinas, J. J. Sáenz, R. Gómez-Medina, and L. Chantada, Opt. Express 18, 11428 (2010).

[34] R. Gómez-Medina, L. S. Froufe-Pérez, M. Yépez, F. Scheffold, M. Nieto-Vesperinas, and J. J. Sáenz, Phys. Rev. A 85, 035802 (2012).

[35] A. Rahimzadegan, R. Alaee, I. Fernandez-Corbaton, and C. Rockstuhl, Phys. Rev. B 95, 035106 (2017).

[36] R. Alaee, M. Albooyeh, and C. Rockstuhl, J. Phys. D 50, 503002 (2017).

[37] I. Fernandez-Corbaton, X. Zambrana-Puyalto, N. Tischler, X. Vidal, M. L. Juan, and G. Molina-Terriza, Phys. Rev. Lett. 111, 060401 (2013).

[38] S. Tretyakov, Analytical Modeling in Applied Electromagnetics (Artech House, Massachusetts, 2003). 
[39] K. J. Webb and A. Ludwig, Phys. Rev. B 78, 153303 (2008).

[40] K. J. Webb and Shivanand, Phys. Rev. E 84, 057602 (2011).

[41] G. W. Mulholland, C. F. Bohren, and K. A. Fuller, Langmuir 10, 2533 (1994).

[42] F. G. De Abajo, Rev. Mod. Phys. 79, 1267 (2007).

[43] I. Fernandez-Corbaton, S. Nanz, R. Alaee, and C. Rockstuhl, Opt. Express 23, 33044 (2015).

[44] R. Alaee, C. Rockstuhl, and I. Fernandez-Corbaton, Opt. Commun. 407, 17 (2018).

[45] M. Safari, M. Albooyeh, C. R. Simovski, and S. A. Tretyakov, Phys. Rev. B 97, 085412 (2018).
[46] R. Duggan, M. A. Miri, and A. Alu, in 2017 IEEE International Symposium on Antennas and Propagation USNC/URSI National Radio Science Meeting (2017), pp. 1067-1068.

[47] P. Yeh, Optical Waves in Layered Media (Wiley-Interscience, New Jersey, 2005), Vol. 61.

[48] L. Ge, Y. D. Chong, and A. D. Stone, Phys. Rev. A 85, 023802 (2012).

[49] G. Hettner, Zeitschrift für Physik 37, 179 (1926).

[50] M. Scandurra, F. Iacopetti, and P. Colona, Phys. Rev. E 75, 026308 (2007). 